\title{
Recomendaciones para Resolución de Urgencias Odontológicas en Atención Primaria de Salud Durante la Pandemia de SARS-CoV-2
}

\author{
Recommendations for the Resolution of Dentistry Emergencies \\ in Primary Health During the SARS-CoV-2 Pandemic
}

Velásquez González Alina ${ }^{1}$; Sepúlveda Verdugo Cristóbal'; Ortuño Borroto Duniel ${ }^{2} \&$ Barrientos Morales Camilo $^{3}$

\begin{abstract}
VELÁSQUEZ, G. A.; SEPÚLVEDA, V. C.; ORTUÑO, B. D. \& BARRIENTOS, M. C. Recomendaciones para resolución de urgencias odontológicas en atención primaria de salud durante la pandemia de SARS-CoV-2. Int. J. Odontostomat., 14(4):548$554,2020$.
\end{abstract}

RESUMEN: La enfermedad originada por el nuevo tipo de coronavirus, SARS-CoV-2, se ha convertido en un problema de Salud Pública a nivel mundial. Esto ha llevado a posponer las atenciones clínicas electivas de pacientes, exceptuando las atenciones de urgencia o emergencia. Las urgencias odontológicas han continuado con alta demanda en el Sistema Público de Salud durante la pandemia COVID-19, e incluso han aumentado en severidad de los cuadros. Las restricciones de horario y las medidas implementadas a nivel país, llevan a que los pacientes consulten en el Centro de Salud más cercano a su domicilio, es decir, Centro de Salud Familiar (CESFAM) o en los Servicios de Atención Primaria de Urgencia (SAPU). Como Cirujanos Dentistas somos parte de un equipo multidisciplinario de salud, por lo que debemos conocer la enfermedad de COVID-19, sus principales signos, síntomas y riesgos asociados. El Cirujano Dentista es indispensable en el diagnóstico, tratamiento y derivación oportuna de enfermedades buco-maxilofaciales, las cuales pueden afectar de forma física y psicológica a los pacientes, llegando a producir complicaciones sistémicas graves si no son tratadas oportunamente. La atención odontológica actual debe incluir el manejo previo, durante y posterior al tratamiento del paciente de APS. Debemos considerar que el personal odontológico tiene un elevado riesgo de transmisión viral, debido a la cercanía con fluidos desde la cavidad oral del paciente. En este artículo se entregaran recomendaciones, basadas en la mejor evidencia disponible y la experiencia clínica actual para la atención odontológica de urgencias enfocadas en la Atención Primaria de Salud.

PALABRAS CLAVE: COVID-19, SARS-CoV-2, Odontología, Salud Pública.

\section{INTRODUCCIÓN}

La pandemia mundial causada por COVID-19 se originó por un nuevo tipo de coronavirus, el cual fue detectado por primera vez en diciembre del 2019, en Wuhan, China. Fue reconocido oficialmente el 8 de enero del 2020, y se ha convertido en un problema de Salud Pública a nivel mundial (Phelan et al. 2020).

El 11 de febrero de 2020 la OMS nombró oficialmente al virus como SARS-CoV-2 "Sindrome Respiratorio Agudo Severo por Coronavirus 2"y la enfermedad causada por este agente viral fue nombrada
COVID-19. Similar al SARS-CoV, reconocido el año 2002 y el Síndrome Respiratorio del Medio Oriente "MERS-CoV" del año 2012. EI SARS-CoV-2 es un virus zoonótico, por tanto se propaga de animales a humanos (Li et al., 2020).

Según el último informe epidemiológico de la Organización Mundial de la Salud (OMS/WHO), a nivel internacional ya son 216 los países que reportan casos de COVID-19, con un total de 12.794 .395 casos confirmados y 566.000 fallecimientos (OMS, 2020).

\footnotetext{
${ }^{1}$ Cirujano Dentista, Servicio de Atención Primaria de Urgencia Dr. Carlos Lorca Tobar, El Bosque, Santiago de Chile, Chile.

${ }^{2}$ Cirujano Dentista, Referente Odontológico, Dirección de Salud Municipal El Bosque, Santiago de Chile, Chile.

${ }^{3}$ Cirujano Dentista, Centro de Salud Familiar Karol Wojtyla, Corporación Municipal de Puente Alto, Santiago de Chile, Chile.
} 
En Chile, hasta el 9 de julio han ocurrido 346.674 casos de COVID-19 (309.274 con confirmación de laboratorio y 37.400 probables, sin confirmación de laboratorio), con una tasa de 1781,6 por 100.000 habitantes. Las mayores tasas de incidencia acumulada por 100.000 habitantes, según casos confirmados por laboratorio se encuentran en la región Metropolitana y es de 3237,9 (Ministerio de Salud, 2020a).

Según la encuesta Casen 2017, un $78 \%$ de la población en Chile pertenece a FONASA (Fondo Nacional de Salud), y en la Región Metropolitana corresponde a un $71,1 \%$ de las personas (Ministerio Desarrollo Social, 2018). En efecto, la mayoría de la población debería asistir a Atención Pública ya sea, primaria, secundaria o terciaria, para obtener una consulta médica gratuita o a bajo costo.

La red de Atención Primaria de Salud (APS) es fundamental en el país, cuenta con equipos multidisciplinarios y abarca población por sectores más reducidos que los servicios de atención de nivel secundario y terciario. Esto es una ventaja, ya que los pacientes que pertenecen a cada Centro de Salud deben recorrer menor distancia para la atención y además se pueden contactar de manera más cercana con el personal de salud.

Las urgencias odontológicas ambulatorias (UOA) comprenden un conjunto de patologías bucomaxilofaciales de aparición súbita y etiología múltiple, que se manifiestan con dolor agudo y/o inflamación, y que provocan una demanda espontánea de atención (Ministerio de Salud, 2020b). Durante el año 2018 se reportaron 346.485 consultas de urgencias odontológicas GES en los establecimientos de la red pública de salud (Ministerio de Salud, 2020c).

El Cirujano Dentista es indispensable en el diagnóstico, tratamiento y derivación oportuna de urgencias y/o emergencias, las cuales, pueden afectar de forma física y psicológica a los pacientes, llegando a producir complicaciones locales, o sistémicas graves si no son tratadas oportunamente.

Es por lo mismo que la atención odontológica de urgencia se ha mantenido sin interrupciones desde el inicio de la pandemia, presentando una continua e incluso aumento de demanda en APS. Los odontólogos se encuentran entre los profesionales con mayor exposición al COVID-19, ya que se realiza un contacto persona a persona, en distancias estrechas y muchos procedimientos generan aerosoles, consi- derando además, que la cavidad oral representa una fuente con alto potencial de transmisibilidad y susceptibilidad a este agente infeccioso (Dar Odeh et al., 2020 Pereira et al., 2020).

Este artículo tiene como objetivo entregar recomendaciones para resolución de urgencias en APS, bajo el contexto de pandemia SARS-CoV-2, según la mejor evidencia disponible y experiencia clínica actual.

\section{Consideraciones previas a atención odontológica}

En el Centro de Salud, se debe realizar un triage previo a la atención, este debe considerar una breve encuesta epidemiológica en relación al COVID-19, con el objetivo de indagar en términos generales síntomas respiratorios y no respiratorios en relación al virus y además posibles contactos con personas diagnosticadas o con sospecha de SARS-CoV-2. Si el paciente presenta síntomas asociados a posible contagio, como son: fiebre mayor a $37,8^{\circ} \mathrm{C}$, tos seca, odinofagia, mialgias, dolor abdominal (Peng et al., 2020), anosmia o ageusia, se deberá derivar a zona estipulada en cada Centro de Salud, donde pueda ser evaluado por el equipo médico.

Es importante además realizar una evaluación odontológica previa a la atención en el box dental. Si hubiese más de un Cirujano Dentista en la jornada o turno, idealmente debe ser realizada por uno de ellos, ya que son los únicos que conocen a cabalidad las urgencias y además para evaluar presencia de fiebre, disfagia y/o odinofagia a causa de infecciones odontogénicas. Se debe explicar a los pacientes, idealmente por medios digitales o teléfonos, que solamente se realizan atenciones de urgencia de acuerdo a GES Odontológico.

\section{Preparación de la Sala de Procedimientos Odontológicos}

En el lugar dónde se realizará la atención odontológica debe mantenerse solamente lo esencial para la resolución de urgencias, de manera de eliminar todos los elementos que puedan contaminarse innecesariamente, si no es posible, se sugiere que deben quedar protegidos con plástico.

La sala de atención debe desinfectarse antes y después de cada paciente. La OMS recomienda la utilización de etanol al $70 \%$ en superficies de menor tamaño. También se ha descrito que la desinfección de superficies con hipoclorito de sodio al $0,1 \%$ ha sido 
eficaz contra coronavirus (Kampf et al., 2020). Se debe considerar la limpieza del sillón dental, salivero, lámpara, las manillas de las puertas, las sillas de operadores, el piso y escritorios, así como también, computadores y teclados correspondientes (Peng et al.). Esto último se recomienda teniendo en cuenta que algunos estudios han reportado la permanencia de SARS-CoV-2 en superficies inanimadas durante tiempos que pueden variar entre dos horas hasta nueve días (Sepúlveda-Verdugo et al., 2020). Además es importante mantener ventilación continua del lugar de atención, En este sentido se recomienda esperar 30 minutos posterior a la atención de pacientes para renovación del aire. No existe evidencia significativa acerca del no uso de aire acondicionado, pero en el caso de utilizarlo, este debe ser en modo de extracción nunca recirculación y cambiar semanalmente los filtros (COVID-19 Dental Services Evidence Review (CoDER) Working Group, 2020).

\section{Preparación del Cirujano Dentista y Técnico en Odontología}

El personal de salud debe prepararse para la atención de pacientes. Previo al uso de elementos de protección personal (EPP), el operador se retirará aros, argollas, reloj, colgantes u otros objetos que puedan entorpecer las distintas etapas de instalación y su uso. Deberá asegurar el cabello a modo de evitar que se desplace sobre su rostro (Ministerio de Salud, 2020c).

\section{Colocación de EPP}

1. Utilizar cubre calzado si existe disponibilidad en el Centro de Salud, de no ser así, utilizar calzado lavable y/o desinfectable.

2. Utilizar un gorro desechable.

3. Realizar lavado de manos clínico, este es considerado uno de los puntos más críticos para reducir la contaminación por partículas virales, debe ser realizado por el profesional antes y después de cada atención (Araya-Salas, 2020).

4. Usar delantal plástico desechable.

5. Uso de mascarilla dependiendo del procedimiento a realizar y del estado del paciente. Se recomienda la utilización de mascarillas quirúrgicas (Center for Disease Control and Prevention, 2020). Es importante destacar que en el caso de que el paciente sea confirmado o con alta sospecha para SARSCoV-2 es recomendable utilizar mascarillas con filtro de partículas FFP2 o tipo N95, por la cercanía del odontólogo a la cavidad oral del paciente, y la posibilidad de tos o estornudos durante el procedimiento, como también está indicado su uso al reali- zar procedimientos odontológicos con generación de aerosoles.

6. Uso de lentes de protección ocular y escudo facial.

7. Utilizar guantes de procedimiento. Existe evidencia en que el uso de doble guante puede conducir a una menor contaminación viral o bacteriana en comparación con un solo guante, pero es baja. Además se limita con que el riesgo de infección aumenta al tener que retirar dos guantes por mano en vez de solo uno (Verbeek et al., 2020).

\section{Preparación del paciente}

El paciente será informado desde que ingresa al Centro de Salud que debe mantener la distancia social mínima de un metro y medio en todo momento. Además se le explicará que debe mantener la mascarilla puesta de manera adecuada y que solo deberá retirarla cuando el personal de salud se lo indique. De esta manera también se evita la propagación del virus de posibles pacientes infectados en la zona de espera. Se debe informar que no debe ingresar al box con ningún elemento en las manos, especialmente celulares para evitar la contaminación cruzada, estos deben estar guardados. Una vez que el paciente ingresa al box odontológico, la historia clínica que el paciente relate debe ser de igual manera con mascarilla y solamente cuando vaya a ser atendido, se solicitará retirarla, manteniéndola el mismo paciente en sus manos.

\section{Atención Odontológica de Urgencia}

En primer lugar, se debe asegurar acceso a las atenciones odontológicas críticas incorporadas en la Garantía Explícita de Salud (GES) de Urgencia Odontológica Ambulatoria, estas son patologías que requieren de atención inmediata, ya sea para manejo de dolor y/o infección, resolución o derivación a centro hospitalario de mayor complejidad de manera oportuna. Se sugiere revisar la nueva Guía de Práctica Clínica como una ayuda en el contexto actual de priorización de urgencias (Ministerio de Salud, 2020b). Las patologías son:
- Pulpitis
- Trauma dentoalveolar (TDA)
- Pericoronaritis
- Infecciones de origen odontogénico
- Abscesos de espacios anatómicos buco- maxilofaciales
- Flegmones
- Gingivitis/Periodontitis ulcero-necrotizante aguda
(GUNA-PUNA)
- Complicaciones post exodoncia 
Además de otorgar atención a las patologías mencionadas, se debe considerar la atención de pacientes oncológicos que requieran tratamiento odontológico de manera inmediata según su condición, como también toma de biopsias en lesiones con sospecha de malignidad.

Los pacientes idealmente deben realizar lavado de manos previo a la atención o higienizar sus manos con alcohol gel al $70 \%$, previo y posterior a la atención dental. Es importante destacar que se sugiere realizar la atención de un paciente por box, aunque exista más de un sillón dental disponible. En la medida de lo posible el paciente debe ingresar sin acompañante al box dental, en caso de menores de edad y pacientes con necesidades especiales, el acompañante debe permanecer con mascarilla durante la atención del paciente (Ministerio de Salud, 2020d).

Una vez en el sillón dental, realizar breve anamnesis, aunque paciente ya haya sido evaluado en triage. Se recomienda realizar preguntar generales de sintomatología, como: ¿Tiene o ha tenido fiebre últimamente?, ¿Ha experimentado recientemente problemas respiratorios como tos o dificultad para respirar? Explicar a los pacientes que si la patología es urgente y requiere resolución inmediata se atenderá igualmente sea positivo o sospechoso para COVID19 , o no. Existe la posibilidad que el paciente haya tenido síntomas y no los relate por temor a no recibir atención dental.

Previo al examen intraoral, se sugiere un enjuague bucal con peróxido de hidrógeno al $1 \%$, el cual se prepara con 1 parte de $\mathrm{H}_{2} \mathrm{O}_{2}$ de 10 volúmenes (o $3 \%$ ) más 2 partes de agua (Ministerio de Salud, 2020d). También se ha descrito el uso de povidona yodada al $0,2 \%$ como colutorio descartando previamente posibles alergias. Se recomiendan estos antisépticos debido a que el SARS-CoV-2 posee susceptibilidad a la oxidación (Peng et al.). Si bien, el enjuague de clorhexidina es el más utilizado en la práctica habitual, puede no ser efectivo para la eliminación de este tipo de virus. Se requieren estudios para evaluar la efectividad de los colutorios bucales en estos contextos.

Se recomienda realizar una anamnesis breve y con la mayor precisión posible con el objetivo de disminuir los tiempos de atención, así como un examen clínico extra e intra-oral. A continuación decidir el tipo de tratamiento que se entregará al paciente, se deben evitar los procedimientos de generación de aerosoles, al igual que el uso de la jeringa triple.
En caso de requerir imágenes de rayos $\mathrm{x}$, la literatura sugiere que se deben priorizar los exámenes extraorales, como radiografía panorámica y cone beam (Vargas-Buratovic et al., 2020). Sin embargo, sabemos que son exámenes más costosos y menos accesibles para los pacientes en APS. Es por esto que recomendamos solicitar únicamente cuando sea indispensable para continuar la atención.

Respetar la delimitación de áreas es una obligación dentro de la atención. No se debe generar contaminación cruzada en el área administrativa y clínica, es importante considerar el uso de distintos materiales de escritorio, como lápices; y la desinfección de ellos al realizar la firma del GES y de consentimientos informados para los procedimientos elegidos como terapia.

De ser posible y acorde a disponibilidad, se sugiere utilizar goma dique en cualquier procedimiento que genere aerosoles, acompañado de un eyector de saliva y de trabajo a cuatro manos (odontólogo-asistente). Ante a la necesidad de realizar una exodoncia, y requerir sutura, se sugiere utilizar de materiales reabsorbibles para disminuir la necesidad de controles (Vargas-Buratovic et al.). Para pacientes con heridas faciales de tejidos blandos, se debe realizar desbridamiento y sutura. En APS es recomendable evaluar posibilidad de realizar restauraciones provisorias y uso de instrumental manual para retiro de caries en resolución de urgencias, como por ejemplo, técnica de restauración atraumática (ART).

En el sistema público de salud se ha mantenido una alta demanda para atención odontológica de urgencia en tiempos de pandemia SARS-CoV-2, y hasta un aumento frente a la ausencia o interrupción de tratamientos odontológicos electivos debido a las indicaciones del MINSAL; lo que ha llevado al aumento de tratamientos mutilantes como las exodoncias (Sigua-Rodríguez et al., 2020) en Atención Primaria. El Cirujano Dentista debe conocer los tipos de tratamientos que se pueden otorgar en APS en estos momentos, y manejar los fármacos para tratamiento de dolor y/o infecciones, de manera que pueda realizar una atención resolutiva para el paciente y evitar una segunda atención por complicaciones.

Dentro de la indicación de medicamentos para control de sintomatología, existió una advertencia en el uso de ibuprofeno en el tratamiento del virus SARSCoV-2, debido al aumento de la expresión de la enzima convertidora de angiotensina-2 (ECA-2), que se 
VELÁSQUEZ, G. A.; SEPÚLVEDA, V. C.; ORTUÑO, B. D. \& BARRIENTOS, M. C. Recomendaciones para resolución de urgencias odontológicas en atención primaria de salud durante la pandemia de SARS-CoV-2. Int. J. Odontostomat., 14(4):548-554, 2020.

cree, es la unión del receptor del virus a las células. Sin embargo la recomendación de la OMS en este caso, es usar paracetamol como tratamiento de primera línea, mientras que el ibuprofeno como tratamiento de segunda línea o complementario, ya que no hay evidencia que el uso de AINES tenga relación con desarrollar COVID19 o una forma más severa de la enfermedad (OMS, 2020). También es importante considerar el metamizol oral o intramuscular generalmente disponible en APS, para manejo de dolor agudo. En caso de infecciones de origen odontogénico se deben seguir las indicaciones y directrices de acuerdo a las patologías de origen infeccioso y la utilización de antibióticos, principalmente amoxicilina como primera linea por 7 días, evaluando cada 24 horas. Las personas que no respondan al tratamiento con amoxicilina precozmente (es decir, que persista el compromiso del estado general después de 48 horas), o que estén inmunosuprimidas, con compromiso de espacios anatómicos profundos, con cuadros difusos como celulitis facial o cuando no se haya podido realizar un tratamiento odontológico (tratamiento endodóncico y drenaje de absceso o exodoncia), podrían beneficiarse de usar amoxicilina más ácido clavulánico como primera opción (Ministerio de Salud, 2020c).

En pacientes confirmados o con sospecha para SARS-CoV-2 que requieran atención de urgencia impostergable, debemos evaluar el EPP disponible en cada centro de salud, si no se cuenta con el equipo necesario, se debe derivar al centro de salud más cercano que posea las condiciones adecuadas.

Para la atención de estos pacientes se deben realizar las mismas medidas de bioseguridad descritas anteriormente. En casos sintomáticos COVID-19, priorizar la derivación para tratamiento médico y la atención odontológica en un contexto hospitalario, si es posible.

Dentro de las recomendaciones de atención para pacientes COVID-19 positivos podemos mencionar:

- Programación de la atención odontológica

- Atención como último paciente del turno o jornada.

- Minimizar procedimientos con generación de aerosoles

- En caso de realizar procedimiento con aerosol, se debe utilizar una mascarilla FFP2 o tipo N95 por paciente - Uso de escudo facial

Como personal de salud en APS, además de atención clínica, debemos educar a los pacientes y reforzar conocimientos sobre la situación actual. Los odontólogos somos agentes de la salud, y una de las funciones esenciales de la Salud Pública es colaborar en situaciones de catástrofes y/o pandemias, con la disminución del problema. Es por esto, que se debe informar a las personas en torno a las medidas de prevención emanadas desde la autoridad sanitaria, poniendo especial énfasis en el lavado de manos como principal medida y reforzar medidas de autocuidado como aislamiento social. Orientación sobre signos y síntomas sugerentes de COVID19, informar sobre plataformas digitales ministeriales, comunales y salud responde (Ministerio de Salud, 2020d).

\section{Consideraciones posteriores a la atención odontológica}

Después de los procedimientos odontológicos, debemos considerar, la eliminación de forma correcta del EPP (Center for Desease and Prevention, 2020). Este es un paso crítico para evitar la transmisión del virus, los equipos de salud deben estar capacitados respecto al uso y retiro de EPP, especialmente en el uso de N95 que es reciente.

Se recomienda el lavado de manos por 40-60 segundos con generación de abundante espuma. En caso de que el paciente sea COVID-19 positivo o que se hayan realizado procedimientos con generación de aerosoles, se debe eliminar la mascarilla utilizada. De no ser el caso, las mascarillas se podrán utilizar por el tiempo que indique el fabricante o hasta que se humedezca.

Se deberán desinfectar las superficies nuevamente. En caso de que el paciente sea sospechoso o positivo para SARS-CoV-2 se debe realizar aseo terminal de la sala de atención y esperar por 30 minutos para continuar con la atención del próximo paciente. Si se generaron aerosoles se debe esperar 3 horas para volver a ingresar al box, o utilizar uno diferente (Van Doremalen et al., 2020).

En relación a la información al paciente, siempre se debe explicar claramente las precauciones que debe tener posterior al procedimiento, especialmente en exodoncias, así se evitarán consultas por complicaciones. Además de sugerir que al llegar a su domicilio debe cambiarse de ropa e idealmente ducharse.

Es fundamental realizar un registro de todos los pacientes atendidos durante el día o jornada. Se debe conocer la información personal y clínica relevante, además del procedimiento que se realizó, debido a 
que estos pacientes al asistir a un Centro de Salud tienen contacto con más personas y se exponen a posibles contagios. Se ha recomendado que si algún paciente se notifica como caso en los 2 días posteriores a la atención, debe contactar al centro para realizar un seguimiento.

\section{CONCLUSIONES}

Debido a la dificultad de trazabilidad que existe actualmente, todas las medidas de protección deben ser utilizadas en los pacientes como si fueran casos sospechosos o probables. De esta manera se evita al máximo contagios de pacientes asintomáticos, que comienzan con los síntomas en días posteriores a la atención o aquellos que estaban en su periodo de infección activo.

Es de suma importancia que el Cirujano Dentista que se desempeña en APS pueda manejar a cabalidad las características y el uso del EPP que maneja cada Centro de Salud para el área odontológica, ya que muchas veces estos recursos son limitados. Así también, es igual de relevante reconocer sus propias limitaciones para la resolución y por tanto realizar derivación de UOA de manera oportuna y eficiente.

Debido a la alta demanda en el Sistema Público de Salud existen muchos pacientes en APS a los cuales se ha pospuesto los controles odontológicos, por lo que una vez superada la contingencia, los equipos deberán priorizar y garantizar la continuidad de la atención a todas las personas con atenciones odontológicas no efectivas durante este periodo (Ministerio de Salud, 2020d).

Debemos considerar que estas medidas para la atención se tendrán que mantener durante un tiempo extenso y como personal de salud es nuestro deber cumplir con las máximas medidas de precaución posibles y educar a la población, para así evitar contagios en el personal de salud y en los pacientes, además de posibles rebrotes de SARS-CoV-2 en un futuro.

Finalmente se debe considerar que las recomendaciones contenidas pueden sufrir variaciones debido a que la evidencia disponible es dinámica en el contexto epidemiológico actual.
VELÁSQUEZ, G. A.; SEPÚLVEDA, V. C.; ORTUÑO, B. D. \& BARRIENTOS, M. C. Recommendations for the resolution of dentistry emergencies in primary health during the SARS-CoV-2 pandemic. Int. J. Odontostomat., 14(4):548-554, 2020.

ABSTRACT: The disease caused by the new coronavirus, SARS-CoV-2 has become a world wide public health problem. This has lead to pospone elective clinical care, maintaining urgent and emergency care. Dental emergency maintains high demand, even more severe clinical situations, at the public health system during the pandemic COVID-19. The local restrictions implemented makes patients to consult at the closest health center, like the Family health centers (CESFAM) or at primary health urgency centers (SAPU). As dental surgeons, part of the multidisciplinary health team, it is a duty to know the COVID19, signs, simptoms and associated risks. The dental surgeon it is essential in the diagnosis, traetment and timely referral of maxilofacial diseases, wich can affect patients physically and psychologically, even leading to serious systemic complications if not treated promptly. Dental care should have previous, during and posterior considerations in pandemic situations. Dental staff has high viral transmisión risk, due to closeness with oral cavity fluids. This article will provide evidence based recommendations and current clinical experience for dental emergency care at primary health centers.

KEY WORDS: COVID-19, SARS-CoV-2, Dentistry, Public Health.

\section{REFERENCIAS BIBLIOGRÁFICAS}

Araya-Salas, C. Considerations for emergency dental care and preventive measures for COVID-19 (SARS-CoV 2). Int. J. Odontostomat., 14(3):268-70, 2020.

Centers for Disease Control and Prevention. Coronavirus Disease. Your Health. How It Spreads. Washington D. C., U. S. Department of Health \& Human Services, 2020. Disponible en: https:// www.cdc.gov/coronavirus/2019-ncov/about/transmission.html

COVID-19 Dental Services Evidence Review (CoDER) Working Group. Recommendations for the Re-Opening of Dental Services: A Rapid Review of International Sources. Cochrane Oral Health, 2020. Disponible en: https://oralhealth.cochrane.org/sites/ oralhealth.cochrane.org/files/public/uploads/ covid19_dental_review_16_may_2020_update.pdf

Dar Odeh, $\bar{N}$.; Babkair, H.; Abu-Hammad, S.; Borzangy, S.; AbuHammad, A. \& Abu-Hammad, O. COVID-19: present and future challenges for dental practice. Int. J. Environ. Res. Public Health, 17(9):3151, 2020.

Kampf, G.; Todt, D.; Pfaender, S. \& Steinmann, E. Persistence of coronaviruses on inanimate surfaces and their inactivation with biocidal agents. J. Hosp. Infect., 104(3):246-51, 2020.

Li, J. Y.; You, Z.; Wang, Q.; Zhou, Z. J.; Qiu, Y.; Luo, R. \& Ge, X. Y. The epidemic of 2019-novel-coronavirus (2019-nCoV) pneumonia and insights for emerging infectious diseases in the future. Microbes Infect., 22(2):80-5, 2020. 
VELÁSQUEZ, G. A.; SEPÚLVEDA, V. C.; ORTUÑO, B. D. \& BARRIENTOS, M. C. Recomendaciones para resolución de urgencias odontológicas en atención primaria de salud durante la pandemia de SARS-CoV-2. Int. J. Odontostomat., 14(4):548-554, 2020.

Ministerio de Desarrollo Social. Encuesta CASEN 2017. Santiago de Chile, Ministerio de Desarrollo Social, Gobierno de Chile, 2018. Disponible en:

http://observatorio.ministeriodesarrollosocial.gob.cl/casenmultidimensional/casen/casen_2017.php

Ministerio de Salud. Informe Epidemiológico $N^{\circ} 32$. Enfermedad por SARS-CoV-2 (COVID-19). Chile 10-07-2020. Santiago de Chile, Departamento de Epidemiología, Ministerio de Salud, Gobierno de Chile, 2020a. Disponible en: https://www.minsal.cl/wpcontent/uploads/2020/07/InformeEpi110720.pdf

Ministerio de Salud. AUGE. 46. Urgencia Odontológica Ambulatoria. Santiago de Chile, Ministerio de Salud, Gobierno de Chile, 2020b. Disponible en: https://auge.minsal.cl/problemasdesalud/index/46

Ministerio de Salud. Guía de Práctica Clínica Urgencias Odontológicas Ambulatorias 2020. Santiago de Chile, Ministerio de Salud, Gobierno de Chile, 2020c. Disponible en: https:// diprece.minsal.cl/garantias-explicitas-en-salud-auge-o-ges/ guias-de-practica-clinica/urgencia-odontologica-ambulatoria/recomendaciones/

Organización Mundial de la Salud (OMS). PAHO COVID-19. Ginebra, Organización Mundial de la Salud, 2020. Disponible en: https://paho-covid19-response-who.hub.arcgis.com/

Peng, X.; Xu, X.; Li, Y.; Cheng, L.; Zhou, X. \& Ren, B. Transmission routes of $2019-n C o V$ and controls in dental practice. Int. J. Oral Sci., 12:9, 2020.

Pereira, L. J.; Pereira, C. V.; Murata, R. M.; Pardi, V. \& PereiraDourado, S. M. Biological and social aspects of Coronavirus Disease 2019 (COVID-19) related to oral health. Braz. Oral Res., 34:e041, 2020.

Phelan, A. L.; Katz, R. \& Gostin, L. O. The novel coronavirus originating in Wuhan, China. Challenges for global health governance. JAMA, 323(8):709-10, 2020.

Sepúlveda-Verdugo, C.; Secchi-Álvarez, A. \& Donoso-Hofer, F. Urgency dental care considerations in the context of coronavirus COVID-19 (SARS-CoV-2). Int. J. Odontostomat., 14(3):279-84, 2020.

Sigua-Rodríguez, E. A.; Bernal-Pérez, J. L.; Lanata-Flores, A. G.; Sánchez-Romero, C.; Rodríguez-Chessa, J.; Haidar, Z. S.; Olate, S. \& Iwaki-Filho, L. COVID-19 and dentistry: a review of recommendations and perspectives for Latin America. Int. J. Odontostomat., 14(3):299-309, 2020.

Subsecretaría de Salud Pública. Orientaciones para Atención Odontológica en Fase 4 COVID-19. Santiago de Chile, Subsecretaría de Salud Pública, División de Prevención y Control de Enfermedades, Departamento Salud Bucal, Ministerio de Salud, Gobierno de Chile, 2020. Disponible en: https:// diprece.minsal.cl/wp-content/uploads/2020/03/ORIENTACIONES-ATENCION-ODONTOLOGICAS-COVID-19-.pdf

Van Doremalen N, Morris DH, Holbrook MG, Gamble A, Williamson BN, et al. Aerosol and Surface Stability of SARS-CoV-2 as Compared with SARS-CoV-1. NEJM March 17, 2020. doi: 10.1056/NEJMc2004973

Vargas-Buratovic, J. P.; Verdugo-Paiva, F.; Véliz-Paiva, C.; LópezTagle, E.; Ahumada-Salinas, A. \& Ortuño-Borroto, D. Dental recommendations in the COVID-19 pandemic: a narrative review. Medwave, 20(4):e7916, 2020.

Verbeek, J. H.; ljaz, S.; Mischke, C.; Ruotsalainen, J. H.; Mäkelä, E.; Neuvonen, K.; Edmond, M. B.; Sauni, R.; Balci, F. S. K. \& Mihalache, R. C. Personal protective equipment for preventing highly infectious diseases due to exposure to contaminated body fluids in healthcare staff. Cochrane Database Syst. Rev., 4:CD011621, 2020
Dirección para correspondencia:

Alina Velásquez González

SAPU Dr. Carlos Lorca

Alfalfal 634

El Bosque

Santiago

CHILE

Email: alina.rvg@gmail.com

Recibido : 13-07-2020

Aceptado: 20-07-2020 